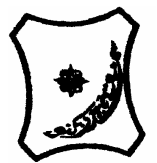

Bayero Journal of Pure and Applied Sciences, 4(2): 80-82

Received: February, 2011

Accepted: September, 2011

ISSN $2006-6996$

\title{
EVALUATION OF ANTHROPOMETRIC STATUS OF HAUSAS OF NORTHERN NIGERIA
}

\author{
*Taura M.G. \\ Department of Anatomy, Faculty of Medicine, Bayero Univeristy, Kano, Nigeria \\ *Correspondence author: mg_taura@yahoo.com
}

\begin{abstract}
Anthropometry is a branch of anthropology that deals with the measurements of various parts of human body which are expected to grow predictably and proportionately. The aim of this work was to study some of the anthropometric parameters of the Hausa people residing in Kano, northwestern Nigeria. Anthropometric parameters measured and recorded in this study were height, weight, body mass index and blood pressure. The measurements were made according to the standard procedures. Sample size of 1113 subjects comprising of $599(53.8 \%)$ males and 504 (46.2\%) females were used for the study. The age range of the subjects was 18 to 60 years with mean age of 23.2 years. Instruments used included weighing machine, stadiometer, and blood pressure apparatus. The results showed that all values were higher in males than females except for body mass index which was higher in female subjects. Bivariate analysis showed a positive correlation between the parameters except for height and body mass index where a negative correlation was observed. The values of the anthropometric parameters in this study were similar to those of other Nigerian ethnic groups and tribes. The values also correlate with the findings from other countries. It is concluded from the findings of this study that the anthropometric parameters and blood pressure of the Hausas of northern Nigeria are within acceptable reference range.
\end{abstract}

Keywords: Anthropometry, parameters, Hausas, Nigeria

\section{INTRODUCTION}

Anthropometry is a branch of anthropology which deals with measurements of different parts of human body. It provides the single most portable, universally applicable, inexpensive and non-invasive technique for assessing the size, proportions and composition of the human body. It also reflects both health and nutritional status and predicts performance, health and survival. For convenience of description, the human body has been described as having regions and sub-regions. These various parts of the human body are expected to grow predictably and proportionately. The relationship of one part of the human body to the other is expected to be constant at specific ages. These relationships are expressed as ratios, indices, or by the use of regression techniques and they change dramatically from the fetal period through childhood to adolescence. This is because of the various interactions between genetic, biochemical and environmental factors.

$$
\text { Generally variations measured in }
$$
anthropometry are those controlled by polygenic inheritance and other factors such as age, sex and nutritional status. Thus the simplest of the parameters in anthropometry include height, weight, length, thickness and width of various parts of the body which vary with different tribes and races (Ferro et.al 1993). Height and weight are the most commonly utilized but not the only anthropometric indicators of nutritional status and health. Human height is the measurement of the length of the human body from the bottom of the feet to the vertex of the head when standing erect. When populations share genetic background and environmental factors, average height is frequently variable within such a population. In regions of extreme poverty or profound warfare, environmental factors like malnutrition during childhood or adolescence may account for marked reductions in adult stature even without the presence of any medical conditions like gigantism or dwarfism. Differences in height within individuals and population could be due to genetic variability or adulthood lifestyle. Some of the tallest people recorded in history include Leonical Stadnyk of Ukraine $(2.57 \mathrm{~m})$, Sultan Kosen of Turkey $(2.47 \mathrm{~m})$, Bao Xishun of Mongolia (2.36 m) and Robert Pershing Wadlow from Illiois in the United States born in 1918 and at the time of his death in 1940 his height was $2.72 \mathrm{~m}$. Epidemiological studies have also demonstrated a positive correlation between height and intelligence (Billy and Benny 2010). Historically this assumption has not always reflected reality. For instance Napoleon and Alexander the Great were not taller than $1.5 \mathrm{~m}$ according to many sources. In sports like basket ball height can be an advantage where the average height of the players is about $1.98 \mathrm{~m}$. Other sports where height can be an advantage or disadvantage include football, cricket, rugby and many more.

Being overweight or underweight can cause changes in human body shape as well as posture and walking style. Obesity is measured using body mass index (BMI) or waist circumference. Body mass index has been conveniently used to define and classify over weight and obesity. It is an objective scientific measure which uses height and weight to determine thinness or overweight. It is calculated as weight in kilogram divided by the square of height in meter. 
According to WHO classification the normal range of BMI is from 18.50-24.99 (Ferro et al., 1993). A BMI of 25.00 to 29.99 is classified as grade I over weight, 3039.99 grade 2 overweight and BMI of 40 and above grade 3 over weight. Recent classification by National Heart, Lung and Blood Institute (1998) stated that a BMI ranging between 25.6-29.9 is over weight and 30 and above is obese. From the results of this study about $95 \%$ of the subjects had a BMI of between 18.0 to 25.0 with a mean BMI of $22.22 \pm 3.36$ and $23.06 \pm$ 4.59 in males and females respectively. Depending on the BMI a person may be referred to as slim, overweight or obese. Excessive body weight is associated with various diseases particularly cardiovascular diseases, diabetes mellitus and certain types of cancer (Allison et al, 1999). Obesity is still seen as a sign of wealth and well being in many parts of Africa. This has become particularly common since the HIV epidemics began (Gray 2004). It was predicted that overweight and obesity may soon replace more traditional public health problem such as malnutrition and infectious diseases. Measurements of height are relatively easy to make as are the measurements of body weight. Body weight of an individual is influenced by the person's height and it rises with increase in status if normal body proportions are maintained. It is a common knowledge that BMI correlates with body fat. The relationship between fatness and BMI differs with age and gender for example; women are more likely to have a higher percentage of body fat than men for the same BMI (Ferro et al., 1993). A systematic review of longitudinal observational studies confirmed a positive association between birth weight and BMI in both children and adults (Atiku et al., 1997). However, studies between BMI and obesity among older adults show that obesity is a major risk factor for cardiovascular diseases, diabetes mellitus, hypertension and premature death and is increasing in the industrialized countries (Deurenberg et al., 2001). As BMI increases the risk of certain diseases may also increase. Some common conditions related to overweight and obesity include premature death, cardiovascular diseases, osteoarthritis, cancer and diabetes mellitus (Seidel 1995). It is reported that BMI is only one of the many factors used to predict risk for diseases (McGarvey and Baker, 1979).

Blood pressure (BP) levels of a population are influenced by a variety of biological, behavioral

and socio-economic factors and show some variations (Fleming et.al.1991). It is one of the most important factors which determine health and activities of other body systems. It is widely accepted that the blood pressure of $120 / 80 \mathrm{~mm}$ is ideal for the adults (Daniel et al., 1996). This tends to increase with advancing age and generally higher in males than in females. The aim of this study was to evaluate blood pressure and anthropometric status of height, weight, blood pressure and body mass index of Hausa people of northern Nigerian Hausa population with a view to comparing these values with the data from other tribes and races.

\section{MATERIALS AND METHODS}

This is a cross-sectional study of height, weight, body mass index and blood pressure on Hausas of northern Nigeria conducted at Bayero University, Kano, Nigeria. Subjects used were consented adult male and female Hausas by identity who were apparently healthy with absence of congenital malformations or external deformities. Overtly sick and clinically sick individuals were excluded in this study.

The sample size consisted of 1113 subjects randomly selected from the Hausa people of northern Nigeria residing in Kano State. Age range of the subjects was 18 to 60 years. Height, weight, age, blood pressure, sex and state of origin were obtained and recorded among others. The data were analyzed using statistical packages for the social sciences (SPSS) to express the descriptive statistics. Students' t-test was used to find whether differences exist between values from males and females subjects. Correlation analysis was performed using Pearson's linear correlation analysis. Body mass index (BMI) was calculated as an index of weight in kilogram divided by height in meter square.

\section{RESULTS}

Table 1 shows the mean and standard deviation of the anthropometric variables measured in male and female subjects. The mean values of height, weight, age and blood pressures were significantly greater in the male than in the female subjects $(p<0.05)$. Body mass index was however greater in females than males ( $p<0.05)$.

All the variables had a positive correlation with one another except height and body mass index which had a negative correlation $(p<0.01)$ as shown in Table 2.

Table 1: Height, Weight, Blood Pressure and Body Mass Index of Hausas of northern Nigeria Males $(n=599)$ Females $(n=504)$

\begin{tabular}{lcll}
\hline Variable & & & \\
\hline HT $(\mathrm{M})$ & $1.69 \pm 0.082$ & $1.60 \pm 0.068$ & 0.05 \\
WT $(\mathrm{kg})$ & $62.09 \pm 10.69$ & $59.07 \pm 12.29$ & 0.05 \\
BMI $(\mathrm{kg} / \mathrm{m} 2)$ & $21.77 \pm 3.39$ & $23.06 \pm 4.75$ & 0.05 \\
Age $($ years $)$ & $23.70 \pm 4.81$ & $22.74 \pm 4.72$ & 0.05 \\
SBP $(\mathrm{mm} \mathrm{Hg})$ & $116.87 \pm 10.09$ & $111.69 \pm 9.82$ & 0.05 \\
DBP $(\mathrm{mm} \mathrm{Hg})$ & $77.57 \pm 8.01$ & $73.85 \pm 8.17$ & 0.05 \\
\hline
\end{tabular}

Key: values are mean \pm standard deviation, Differences were statically significant in the above parameters at $p<$ 0.05, S.D. - Standard Deviation, HT - Height, WT - Weight, BMI - Body mass index, SBP - Systolic Blood Pressure, DBP - Diastolic Blood Pressure 
Table 2: Bivariate analysis of Anthropometric Variables of Hausas of northern Nigeria

\begin{tabular}{lcc}
\hline Variables & Correlation & P. Value \\
\hline HT and WT & 0.43 & 0.01 \\
HT and BMI & 0.12 & 0.01 \\
WT and BMI & 0.83 & 0.01 \\
Age and BMI & 0.32 & 0.01 \\
WT and SBP & 0.26 & 0.01 \\
WT and DBP & 0.23 & 0.01 \\
BMI and SBP & 0.18 & 0.01 \\
BMI and DBP & 0.16 & 0.01 \\
SBP and DBP & 0.69 & 0.01 \\
\hline
\end{tabular}

HT - Height, WT - Weight, BMI - Body Mass Index, SBP - Systolic Blood Pressure, DBP - Diastolic Blood Pressure $(P<0.01)$

\section{DISCUSSION}

The anthropometric values obtained from this study were similar to those from other studies within Nigeria and some African countries. In all the parameters measured males had higher values than females except for body mass index which was higher in females than in males. The values for height were slightly lower compared to what was obtained on Nigerians residing in Jos Plateau State but higher than those of the Igbos of Eastern Nigeria (Ekezie 2006)

The mean height of the subjects obtained in the present study agrees with the values obtained in Australian males and females. This shows that Hausa men are slightly taller than Australian men, while the Australian women are taller compared to Hausa women (Ferro et. al 2003). When compared with subjects from Brazil, the Hausas of northern Nigeria had a greater mean height for both males and females. On the other hand the Hausa people of Northern Nigeria had a lower mean height compared to the citizens of the United States (Calle et al., 1999). In conformity to the usual trend the result of this study showed a higher value of weight for males compared to females which follows the same trend with the previous studies. The mean weight obtained from this study is lower than the values obtained on male Kenyans and female Kenyans (Taura. 2009). The value could also be compared with values obtained from United

\section{REFERENCES}

Allison D.B., Fontaine K. R., Manson J. E., Stevens J and Vantallie T B (1999) : Annual death attributable to obesity in the United States JAMA 282 (16) 1530-8

Atiku, M.K., Sen, K.K. and Temple, V.J. (1997): Age and sex distribution of body mass index and body surface area among Nigerians. Proceedings of the $28^{\text {th }}$ Annual Conference, Nutrition Society of Nigeria. Bayero University, Kano 81-82.

Billy and Benny McCrary (2010), Holders of Guinness World Record for World's Heaviest Twins (Medline Search)

Calle EE, thum MJ, Petrlli JM. Rodrigues C, health CW (1999) Body mass index and mortality in a prospective cohort of US adults N. Engl J Med 7:341 (15) 1140-1.

Daniel, S.R., Orbarzanek C. and Barton, B.A. (1996): Sexual maturation and racial differences in blood pressure in girls. The National, Heart, Lung and Blood Institute Growth and study. I Paediatric 129: (208-213)

Deurenberg, Y. M, Chew, S.K., Tan, V.F., Van, B.Y. and Staveren, W.A (2001) Relationships between indices of obesity and its co-mobidities in multiethnic Singapore Int. J. Obes. Relat. Metab. Discord; 25;155-162.
State of America with male U. S. citizens (Calle et al., 1999).

With the exception of few subjects, most of the subjects recruited for the present study had their blood pressure and anthropometric parameters within the reference range. These suggest that Hausas people of northern Nigerian origin have an ideal blood pressure and metabolic profile and therefore not at risk of developing cardiovascular diseases and other related systemic diseases. The significant differences in some areas as observed in the present study could be due or environmental factors.

\section{CONCLUSION AND RECOMMENDATION}

Anthropometric parameters and blood pressure of the Hausas are within the reference range and are comparable to those obtained from other Nigerian tribes. The values for these parameters were equally comparable to those obtained from citizens of other nations, ethnic groups and other races.

It is recommended that racial and ethnic backgrounds should be considered in making clinical or anatomical judgments of the people. It is recommended that further studies be carried out to determine reference values of these parameters on other Nigerian tribes and ethnic groups.

Dressler, W.W., Greth, G.A, Gallazher, P.N and Viteri F.E (1992). Social Factors mediating social class differences in blood pressure in Jamaican Community. Soc. Sci.Med.35:1233-1244.

Ekezie J. (2006). The effect of sex, marital status, education and exercise on adiposity and blood pressure of Igbos M.Sc. thesis Ahmadu Bello University, Zaria Nigeria.

Ferrol, L.A., Hass, J. and Ransome K. O. (1993). WHO Expert Committee on Physical Status The Use and Interpretation of Anthropometry Geneva 1-38.

Fleming, U.M, Sutos, R.V. and Gimbra, E. (1991). Blood pressure level of the Surni and zoro Indians of the Brazilian Amazon Group and sex specific effects resulting from body composition, health status and age. Hum. Bio. 63:835-861.

Gray G. A. (2004). Medical consequences of obesity J. Clin. Endocrinology metab 89 (6) 2583-9

McGarvey, T. and Baker, P.T (1979). The effects of modernization and migration on Samoans blood pressure. Hum Bio. 51(461-479).

Seidel, J.C, (1995): Obesity in Europe; Scalling and epidemic Int. J Obese (1) 19 Suppl 51-59

Taura M.G. (2009). Anthropometric Studies of Height, Weight and Body Mass Index among Two East African Population groups. Nigerian Journal of Basic and Clinical Sciences (6) 2, 67-72. 